\title{
Lyapunov Analysis of the Chaotic Colpitts Oscillator
}

\author{
Ivana Vasiljević*, Aleksandra Lekić*, Dušan Stipanović ${ }^{\dagger}$ \\ ${ }^{*}$ Department of Electronics, School of Electrical Engineering, University of Belgrade, 11000 Belgrade, Serbia \\ ${ }^{\dagger}$ Coordinated Science Laboratory and ISE Department, University of Illinois at Urbana-Champaign, \\ Urbana, Illinois, USA
}

\begin{abstract}
In this paper, chaotic behavior of the Colpitts oscillator is studied and analyzed. The analyses consider the locally unstable behavior based on the linearization around the equilibrium and the globally bounded stability, which guarantees that the trajectories converge to an attractive and bounded set. The attractive set for the Colpitts oscillator is determined and characterized using Lyapunov analyses and an appropriate quadratic Lyapunov function. The theoretical results are accompanied by a representative simulation.
\end{abstract}

Index Terms-Colpitts oscillator, Lyapunov analysis, chaos.

\section{INTRODUCTION}

It is well known that chaos is a common behavior for the ocillators realized in the microstrip technology as documented in [1]. What is missing is more theoretical analysis type of results that would provide a characterization of this complex and still largely unknown behavior. Certainly some earlier worthy works to mention are related to chaos in circuits [2], [3] as well as related to bifurcations in [4]-[6]. This observation is a motivation for the work presented in this paper. In particular, in this paper we consider the standard Colpitts oscillator [4] [10] and analyze its chaotic behavior. Colpitts oscillator is well known for producing chaotic behaviors and thus it can be used for building chaotic radars and chaotic synchronized systems [9]-[11]. There has also been work that investigated chaotic behavior of these oscillators using Lyapunov analysis [12]. In this paper, we also use Lyapunov analysis to characterize a bounded set in which the oscillator has chaotic behavior. This is done by constructing a quadratic Lyapunov function and showing that the oscillator is globally stable with respect to a bounded set. The bounded set is attractive and characterized as a level set of the corresponding Lyapunov function. On the other hand we show that the equilibrium of the system is unstable and being inside the attractive set it is well aligned with the chaotic behavior. We also provide a way to bound the set away from the equilibrium using the Lyapunov analysis for instability. Despite the fact that outer and inner bounds are constructed using appropriate quadratic Lyapunov functions that are known for not providing tight bounds, in general, we believe that the approach is novel in providing a technically sound characterization of the chaotic behavior in terms of Lyapunov functions rather than exponents that are much more difficult to compute [13]. The next step to be taken is to provide characterization using piecewise linear Lyapunov functions that are known for providing tighter approximations.
This paper is organized as follows. Section II contains model of the Colpitts oscillator used in the paper. In section III, we provide an analysis of the instability of the linearized oscillator equations. Section IV contains a derivation and computation of the Lyapunov function and the related set of attraction characterized in terms of a value set of this Lyapunov function. This section also contains a Lyapunov analysis of instability around the equilibrium, thus, providing another bound for the set where the chaotic behavior is contained. Furthermore, a simulation illustrating the analyses and the chaotic behavior of the oscillator is provided. Finally, some concluding remarks are formulated in section V.

\section{MOdEL DERIVATION}

Colpitts oscillator, as depicted in Fig. 1, is analyzed as a nonlinear circuit with the bipolar junction transistor (BJT) modelled using the standard large signal model. This, as a dynamical system, can be described using three differential and one nonlinear algebraic equation, as follows:

$$
\begin{aligned}
& \frac{d v_{C 1}}{d t}=\frac{1}{C_{1}}\left(i_{L}-\beta i_{B}\right), \\
& \frac{d v_{C 2}}{d t}=\frac{1}{C_{2}}\left(-\frac{v_{C 2}}{R_{E}}+i_{L}+i_{B}-\frac{V_{2}}{R_{E}}\right), \\
& \frac{d i_{L}}{d t}=\frac{1}{L}\left(-v_{C 1}-v_{C 2}-R i_{L}+V_{1}\right), \\
& i_{B}=\frac{I_{S}}{\beta}\left(e^{-\eta \frac{v_{C 2}}{V_{T}}}-1\right) .
\end{aligned}
$$

In equation (1), capacitors' voltages and the inductor current represent state variables of the system. Vector of the state variables is then given as $\mathbf{x}=\left[\begin{array}{lll}v_{C 1} & v_{C 2} & i_{L}\end{array}\right]^{\top}$.

Dynamical system (1) can be rewritten in compact matrix form:

$$
\dot{\mathbf{x}}=\mathbf{A} \mathbf{x}+\mathbf{B}+\mathbf{C} e^{-\eta \frac{x_{2}}{V_{T}}},
$$

where $\mathbf{A}, \mathbf{B}$ and $\mathbf{C}$ are given as

$$
\begin{aligned}
\mathbf{A} & =\left[\begin{array}{ccc}
0 & 0 & \frac{1}{C_{1}} \\
0 & -\frac{1}{R_{E} C_{2}} & \frac{1}{C_{2}} \\
-\frac{1}{L} & -\frac{1}{L} & -\frac{R}{L}
\end{array}\right], \\
\mathbf{B} & =\left[\begin{array}{c}
\frac{I_{S}}{C_{1}} \\
-\frac{V_{2}}{R_{E} C_{2}}-\frac{I_{S}}{\beta C_{2}} \\
\frac{V_{1}}{L}
\end{array}\right], \quad \mathbf{C}=\left[\begin{array}{c}
-\frac{I_{S}}{C_{1}} \\
\frac{I_{S}}{\beta C_{2}} \\
0
\end{array}\right] .
\end{aligned}
$$




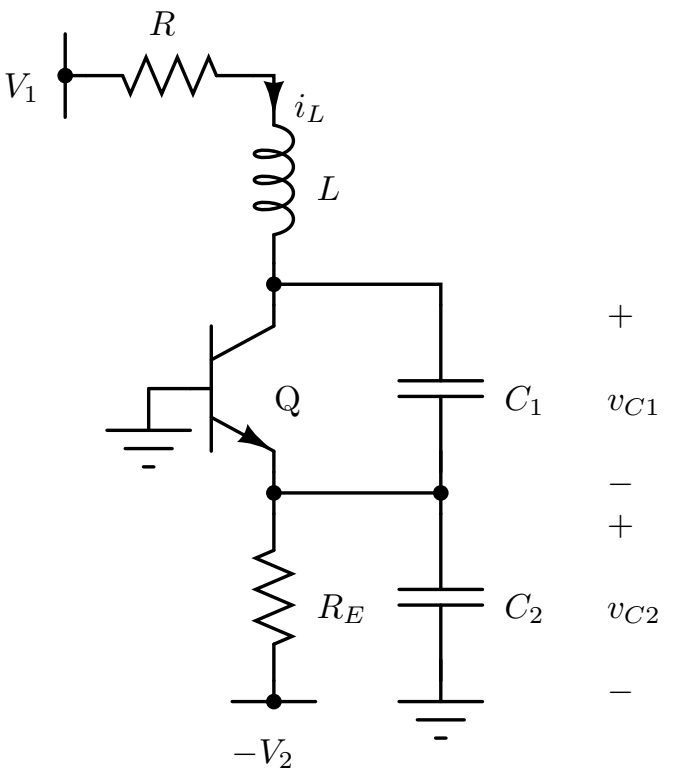

Fig. 1: Colpitts oscillator.

In this paper, the analysis and the simulations are done for the Colpitts oscillator realized using transistor BFU730F with the transistor and circuit parameter given in appendix.

Matrix A (from equation (3)) is computed as stable for the given circuit parameters, which means that it has all eigenvalues with negative real parts. In particular, there is one real eigenvalue $\lambda_{1}=-0.09701 \cdot 10^{9}$ and two complex conjugate ones $\lambda_{2,3}=(-1.0495 \pm 4.3689 i) \cdot 10^{9}$.

\section{LINEARIZATION WITH JACOBIAN MATRIX}

A common and useful way to analyze any systems yet in particular chaotic and unstable systems is to represent them as a sum of linear and nonlinear parts [13]. Along these lines, the system (1) can be described by the following equation:

$$
\dot{\mathbf{x}}=\mathbf{f}(\mathbf{x}),
$$

where function $\mathbf{f}(\mathbf{x})$ can be decomposed as

$$
\mathbf{f}(\mathbf{x})=\mathbf{J} \cdot \mathbf{x}+\mathbf{h}\left(x_{2}\right),
$$

where $\mathbf{J}$ is the Jacobian matrix of the system (1) computed using its equilibrium $\overline{\mathbf{x}}=\left[\begin{array}{lll}\overline{v_{C 1}} & \overline{v_{C 2}} & \overline{i_{L}}\end{array}\right]^{\top}=\left[\begin{array}{lll}\overline{x_{1}} & \overline{x_{2}} & \overline{x_{3}}\end{array}\right]^{\top}$ and $\mathbf{h}\left(x_{2}\right)$ is the nonlinear part of the system (1). The equilibrium is obtained by solving the following system of equations:

$$
\begin{aligned}
& \overline{x_{1}}=V_{1}+V_{2}-\frac{\overline{x_{3}}}{\beta}\left((1+\beta) R_{E}+\beta R\right), \\
& \overline{x_{2}}=\frac{\overline{x_{3}}}{\beta} R_{E}(1+\beta)-V_{2}, \\
& \overline{x_{3}}=I_{S}\left(e^{-\eta \frac{\overline{x_{2}}}{V_{T}}}-1\right) .
\end{aligned}
$$

We have to note here that the equilibrium set of equations (6) has to be solved numerically.

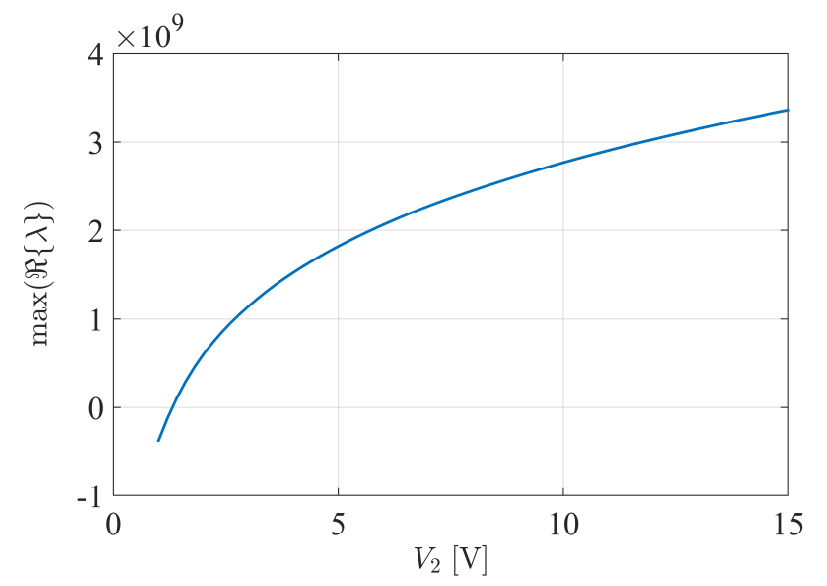

Fig. 2: Dependence of the maximum eigenvalue with respect to the voltage $V_{2}$.

Using system of equations (1) and equilibrium (6), the Jacobian and the nonlinearity are given as

$$
\begin{aligned}
\mathbf{J}= & {\left[\begin{array}{ccc}
0 & \frac{I_{S} \eta}{C_{1} V_{T}} e^{-\eta \frac{\bar{x}_{2}}{V_{T}}} & \frac{1}{C_{1}} \\
0 & -\frac{1}{C_{2} R_{E}}-\frac{I_{S} \eta}{\beta C_{2} V_{T}} e^{-\eta \frac{\bar{x}}{V_{T}}} & \frac{1}{C_{2}} \\
-\frac{1}{L} & -\frac{1}{L} & -\frac{R}{L}
\end{array}\right], } \\
\mathbf{h}\left(x_{2}\right)= & {\left[\begin{array}{c}
-\frac{I_{S}}{C_{1}}\left(e^{-\eta \frac{x_{2}}{V_{T}}}-1\right)-\frac{I_{S} \eta}{C_{1} V_{T}} e^{-\eta \frac{\bar{x}_{2}}{V_{T}}} x_{2} \\
\frac{I_{S}}{\beta C_{2}}\left(e^{-\eta \frac{x_{2}}{V_{T}}}-1\right)-\frac{V_{2}}{C_{2} R_{E}}+\frac{I_{S} \eta}{\beta C_{2} V_{T}} e^{-\eta \frac{\overline{x_{2}}}{V_{T}}} x_{2} \\
\frac{V_{1}}{L}
\end{array}\right] . }
\end{aligned}
$$

Now, the local stability of the linearized system can be determined by the location of the eigenvalues that satisfy the standard characteristic equation given by $\operatorname{det}(\mathbf{J}-\lambda \mathbf{I})=0$, where $\mathbf{J}$ is the Jacobian matrix and $\mathbf{I}$ denotes the identity matrix of the same dimension as the Jacobian. If there is at least one eigenvalue with a positive real part, the system is unstable. From the diagram depicted in Fig. 2, which shows dependence of the maximum eigenvalue or eigenvalues (if not unique) of the linearized system with respect to the voltage $V_{2}$, it is shown that for the voltage $V_{2}>1.309 \mathrm{~V}$ the behavior of the system is clearly unstable, because its largest eigenvalue has a positive real part.

\section{ANALYSIS OF THE BOUNDEDNESS OF THE SYSTEM TRAJECTORIES USING LYAPUNOV APPROACH}

Contrary to the system linearization showing unstable local behavior for the big range of voltage $V_{2}$ 's values, we will show that the system is globally bounded (or stable) for sufficiently large values of the state variables (in terms of their norm values). This will be done by showing that the derivative of a quadratic Lyapunov function is negative when $\|\mathbf{x}\| \geq K$. 
Now, let us choose a commonly used quadratic Lyapunov function as a candidate in the following form:

$$
V(\mathbf{x})=\mathbf{x}^{T} \mathbf{P} \mathbf{x},
$$

where matrix $\mathbf{P} \succ 0$ is a positive definite matrix and the time derivative of Lyapunov function is computed as

$$
\begin{aligned}
\dot{V}(\mathbf{x}) & =\dot{\mathbf{x}}^{\top} \mathbf{P} \mathbf{x}+\mathbf{x}^{\boldsymbol{\top}} \mathbf{P} \dot{\mathbf{x}} \\
& =\mathbf{x}^{\boldsymbol{\top}} \underbrace{\left(\mathbf{A}^{\top} \mathbf{P}+\mathbf{P A}\right)}_{-\mathbf{Q}} \mathbf{x}+\underbrace{2 \mathbf{B}^{\boldsymbol{\top}} \mathbf{P}}_{\mathbf{b}} \mathbf{x}+\underbrace{2 \mathbf{c}^{\top} \mathbf{P}}_{\mathbf{c}} \mathbf{x} e^{-\eta \frac{x_{2}}{V_{T}}} \\
& =-\mathbf{x}^{\boldsymbol{\top}} \mathbf{Q} \mathbf{x}+\mathbf{b} \mathbf{x}+\mathbf{c x} e^{-\eta \frac{x_{2}}{V_{T}}} .
\end{aligned}
$$

In assessing the sign of $\dot{V}(\mathbf{x})$, the following inequalities have been used:

$$
\begin{aligned}
-\mathbf{x}^{\top} \mathbf{Q} \mathbf{x} & \leq-\lambda_{\min }(\mathbf{Q})\|\mathbf{x}\|^{2}, \\
\mathbf{b} \mathbf{x} & \leq\|\mathbf{b}\|\|\mathbf{x}\| .
\end{aligned}
$$

where $\lambda_{\max }(\cdot)$ and $\lambda_{\min }(\cdot)$ present maximum and minimum eigenvalue of the positive definite matrix, respectively. The standard Eucledian norm is denoted as $\|\cdot\|$. Using inequalities (9), an estimate of the time derivative of the Lyapunov function (8) is given by a simplified expression as

$$
\dot{V}(\mathbf{x}) \leq-\lambda_{\min }(\mathbf{Q})\|\mathbf{x}\|^{2}+\|\mathbf{b}\|\|\mathbf{x}\|+\mathbf{c} \mathbf{x} e^{-\eta \frac{x_{2}}{V_{T}}} .
$$

Then, the system is bounded and the trajectories minimies the Lyapunov functions values when the expression for the Lyapunov function time derivative is negative. In order to show this, two cases can be considered depending on the value of variable $x_{2}$.

In the case when $x_{2}>0$, the inequality is satisfied since $e^{-\eta \frac{x_{2}}{V_{T}}<1}$ and

$$
\mathbf{c} \mathbf{x} e^{-\eta \frac{x_{2}}{V_{T}}}<\mathbf{c} \cdot \mathbf{x} \cdot 1 \leq\|\mathbf{c}\|\|\mathbf{x}\| .
$$

Then the Lyapunov function time derivative from equation (10) becomes

$$
\dot{V}(\mathbf{x}) \leq\|\mathbf{x}\| \cdot\left(-\lambda_{\min }(\mathbf{Q})\|\mathbf{x}\|+\|\mathbf{b}\|+\|\mathbf{c}\|\right),
$$

and it is negative when

$$
\|\mathbf{x}\|>K_{1}=\frac{\|\mathbf{b}\|+\|\mathbf{c}\|}{\lambda_{\min }(\mathbf{Q})}
$$

The other case is when $x_{2}<0$ implying $\mathbf{c} \mathbf{x}<0$. Then, it stems that $\mathbf{c} \mathbf{x} e^{-\eta \frac{x_{2}}{V_{T}}}<0$ and from equation (10) it follows that a sufficient condition is given by

$$
\|\mathbf{x}\|\left(-\lambda_{\min }(\mathbf{Q})+\|b\|\right) \leq 0,
$$

which provides the following bound:

$$
\|\mathbf{x}\|>K_{2}=\frac{\|\mathbf{b}\|}{\lambda_{\min }(\mathbf{Q})}
$$

From equations (11) and (12) it follows that the Lyapunov function time derivative is definitely negative if $\|\mathbf{x}\| \geq K=\max \left(K_{1}, K_{2}\right)$.

\section{A. Local instability around the equilibrium analysis}

Since the original system is locally unstable around the equilibrium, we would also like to characterize its instability. In order to do so we can also use Lyapunov analysis for instability [14]. In order to do so we do a standard decomposition of the original system (5) into stable and unstable parts. Therefore, we introduce a change of variables $\mathbf{y}=\mathbf{x}-\overline{\mathbf{x}}$, where equilibrium $\overline{\mathbf{x}}$ is obtained through the equation (6). The new system is

$$
\dot{\mathbf{y}}=\mathbf{J y}+\mathbf{h}_{y}\left(y_{2}\right),
$$

for $\mathbf{h}_{y}\left(y_{2}\right)=\mathbf{J} \overline{\mathbf{x}}+\mathbf{h}\left(x_{2}\right)$.

Now, our goal is decompose the system matrix $\mathbf{J}$ into the stable and unstable parts so that $\mathbf{J}=\mathbf{T} \mathbf{J}_{D} \mathbf{T}^{-1}$, where $\mathbf{J}_{D}$ corresponds to the Jordan form of the matrix $\mathbf{J}$ and nonsingular matrix $\mathbf{T}$ with its columns being eigenvectors. In section III it is shown that matrix $\mathbf{J}$ has two unstable conjugate-complex and one stable eigenvalue, so the matrix $\mathbf{J}_{D}$ has block diagonal form with two unstable and one stable eigenvalues.

If we multiply equation (13) from the left by matrix $\mathbf{T}^{-1}$ and denote $\mathbf{z}=\mathbf{T}^{-1} \mathbf{y}$, the new differential equation is obtained as

$$
\dot{\mathbf{z}}=\mathbf{J}_{D} \mathbf{z}+\mathbf{h}_{z}\left(z_{2}\right) .
$$

Then, the unstable matrix $\mathbf{J}_{D}$ with only strictly stable and unstable eigenvalues implies the corresponding partition of the overall state vector into sub-vectors denoted as $\mathbf{z}$ being $\mathbf{z}=$ $\left[\mathbf{z}_{1}^{\top}, \mathbf{z}_{2}^{\top}\right]^{\top}$. Now, we know that the Lyapunov matrix equation:

$$
\mathbf{J}_{D}^{\top} \mathbf{P}_{D}+\mathbf{P}_{D} \mathbf{J}_{D}=\left[\begin{array}{cc}
-\mathbf{Q}_{1} & 0 \\
0 & -\mathbf{Q}_{2}
\end{array}\right]=-\mathbf{Q}_{D}
$$

has a solution in the form of $\mathbf{P}_{D}=\left[\begin{array}{cc}\mathbf{P}_{1} & 0 \\ 0 & -\mathbf{P}_{2}\end{array}\right]$ for positive definite $\mathbf{P}_{i}, \mathbf{Q}_{i}$, for $i=1,2$. If we partition matrix $\mathbf{J}_{D}$ accordingly, that is, $\mathbf{J}_{D}=\left[\begin{array}{cc}\mathbf{J}_{11} & \mathbf{0} \\ \mathbf{0} & \mathbf{J}_{22}\end{array}\right]$, which implies $\mathbf{J}_{D}^{\top}=$ $\left[\begin{array}{cc}\mathbf{J}_{11}^{\top} & \mathbf{0} \\ \mathbf{0} & \mathbf{J}_{22}^{\top}\end{array}\right]$. Then, the Lyapunov matrix equation becomes

$$
\begin{aligned}
& {\left[\begin{array}{cc}
\mathbf{J}_{11}^{\top} & \mathbf{0} \\
\mathbf{0} & \mathbf{J}_{22}^{\top}
\end{array}\right]\left[\begin{array}{cc}
\mathbf{P}_{1} & 0 \\
0 & -\mathbf{P}_{2}
\end{array}\right]+\left[\begin{array}{cc}
\mathbf{P}_{1} & 0 \\
0 & -\mathbf{P}_{2}
\end{array}\right]\left[\begin{array}{cc}
\mathbf{J}_{11} & \mathbf{0} \\
\mathbf{0} & \mathbf{J}_{22}
\end{array}\right]=} \\
& {\left[\begin{array}{cc}
\mathbf{J}_{11}^{\top} \mathbf{P}_{1}+\mathbf{P}_{1} \mathbf{J}_{11} & \mathbf{0} \\
\mathbf{0} & \mathbf{J}_{22}^{\top} \mathbf{P}_{2}+\mathbf{P}_{2} \mathbf{J}_{22}
\end{array}\right]=\left[\begin{array}{cc}
-\mathbf{Q}_{1} & 0 \\
0 & -\mathbf{Q}_{2}
\end{array}\right]}
\end{aligned}
$$

Now, we know that the nonlinearity in system (14) can be bounded as $\left\|\mathbf{h}_{z}(\mathbf{z})\right\|<c\|\mathbf{z}\|$, when $\|\mathbf{z}\| \leq r$, for some $r$ and $c$. This is because $c$ always exists for some small enough $r$ due to the linearization of a system with continuously differentiable righthand side. Then, we choose a Lyapunov function candidate $V(\mathbf{z})=\mathbf{z}_{2}^{\top} \mathbf{P}_{2} \mathbf{z}_{2}-\mathbf{z}_{1}^{\top} \mathbf{P}_{1} \mathbf{z}_{1}=-\mathbf{z}^{\top} \mathbf{P z}$ and compute 


$$
\begin{aligned}
\dot{V}(\mathbf{z}) & =-\frac{d}{d t}\left(\mathbf{z}^{\top} \mathbf{P} \mathbf{z}\right)=-\mathbf{z}^{\top}\left(\mathbf{J}_{D}^{\top} \mathbf{P}_{D}+\mathbf{P}_{D} \mathbf{J}_{D}\right) \mathbf{z}-2 \mathbf{z}^{\top} \mathbf{P}_{D} \mathbf{h}_{z} \\
& =\mathbf{z}^{\top} \mathbf{Q}_{D} \mathbf{z}-2 \mathbf{z}^{\top}\left[\begin{array}{cc}
\mathbf{P}_{1} & \mathbf{0} \\
\mathbf{0} & -\mathbf{P}_{2}
\end{array}\right]\left[\begin{array}{c}
\mathbf{h}_{1}(\mathbf{z}) \\
-\mathbf{h}_{2}(\mathbf{z})
\end{array}\right] \\
& =\mathbf{z}^{\top} \mathbf{Q}_{D} \mathbf{z}-2 \mathbf{z}^{\top} \mathbf{P}_{D} \mathbf{h}_{z} \\
& \geq \lambda_{\min }\left(\mathbf{Q}_{D}\right)\|\mathbf{z}\|^{2}-2 \lambda_{\max }\left(\mathbf{P}_{D}\right)\|\mathbf{z}\|\left\|\mathbf{h}_{z}\right\| \\
& \geq\left(\lambda_{\min }\left(\mathbf{Q}_{D}\right)-2 c \lambda_{\max }\left(\mathbf{P}_{D}\right)\right)\|\mathbf{z}\|^{2}>0
\end{aligned}
$$

Thus, choosing $r$ small enough so that $c<0.5 \lambda_{\min }\left(\mathbf{Q}_{D}\right) / \lambda_{\max }\left(\overline{\mathbf{P}_{D}}\right)$ for $\overline{\mathbf{P}_{D}}=\left[\begin{array}{cc}\mathbf{P}_{1} & \mathbf{0} \\ \mathbf{0} & \mathbf{P}_{2}\end{array}\right]$, we get that the systems is unstable.

After applying the inverse transformation, a bound on $\|\mathbf{x}\|$ can be determined as $r_{x}=r\|\mathbf{T}\|$.

\section{B. Simulation results}

In order to demonstrate the accuracy of the determined region of attraction of the Colpitts oscillator with already provided circuit parameters in the appendix and with the voltage $V_{2}=14.8 \mathrm{~V}$, the quadratic Lyapunov function is constructed using linear matrix inequalities (LMIs) that resulted in matrices $\mathbf{P}$ and $\mathbf{Q}$ as follows:

$$
\begin{aligned}
& \mathbf{Q}=\mathbf{I}_{3 \times 3}, \\
& \mathbf{P}=10^{-6}\left[\begin{array}{ccc}
0.0028 & -0.0024 & 0.0050 \\
-0.0024 & 0.0027 & -0.0002 \\
0.0050 & -0.0002 & 0.2388
\end{array}\right] .
\end{aligned}
$$

In the case of a stable system, the problem of maximizing $\lambda_{\min }(\mathbf{Q}) / \lambda_{\max }(\mathbf{P})$ is known to be solved for any diagonal matrix $\mathbf{Q}=a \mathbf{I}$, where $a>0$ (see, for example [15]). In our problem matrix $\mathbf{A}$ is stable (see equation (3)) and thus the choice for the matrix $\mathbf{Q}$ is obvious. For the oscillator's globally attractive set, the obtained boundary value is $K=53.6594$. For the simulated case the maximum obtained norm of the state variables is $\|\mathbf{x}\|_{\max }=11.6281$. Thus, the obtained bound is obviously conservative, which is not surprising since the use of a quadratic Lyapunov function does not provide tight bounds, in general. In order to obtain less conservative bound, in future, polytopic or piecewise linear Lyapunov functions will be considered.

For the same Colpitts oscillator, matrices $\mathbf{P}_{D}$ and $\mathbf{Q}_{D}$ are obtained using LMIs in cvx toolbox (integrated in MATLAB) as

$$
\mathbf{P}_{D}=10^{-7} \cdot\left[\begin{array}{ccc}
-0.0052 & -0.0000 & 0 \\
-0.0000 & -0.0052 & 0 \\
0 & 0 & 0.1839
\end{array}\right]=\left[\begin{array}{cc}
-\mathbf{P}_{2} & \mathbf{0} \\
\mathbf{0} & \mathbf{P}_{1}
\end{array}\right]
$$

and

$$
\mathbf{Q}_{D}=\left[\begin{array}{ccc}
3.4772 & 0.0000 & 0 \\
0.0000 & 3.4772 & 0 \\
0 & 0 & 357.0714
\end{array}\right] \text {. }
$$

The value for constant $c$ is computed as $c=0.95 \times$ $0.5 \lambda_{\min }\left(\mathbf{Q}_{D}\right) / \lambda_{\max }\left(\overline{\mathbf{P}_{D}}\right)=8.9807 \cdot 10^{7}$ when $r=1.7404$. $10^{-5}$ in the transformed space and thus $r_{x}=2.4462 \cdot 10^{-5}$

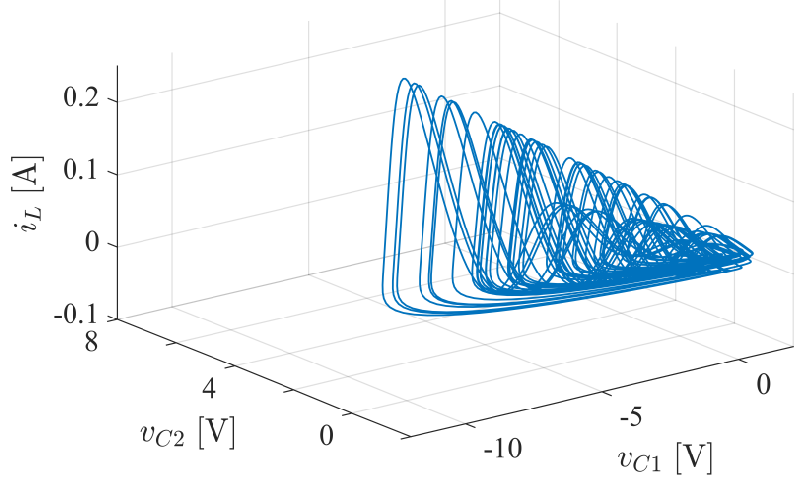

Fig. 3: Phase portrait of the Colpitts oscillator in which is $V_{2}=14.8 \mathrm{~V}$ and the state variables initial values are $\mathbf{x}_{0}=$ $[1.116 \mathrm{~V}-0.665 \mathrm{~V} 27.442 \mathrm{~mA}]^{\top}$.

in the original space. The minimum obtained norm of the state variables around equilibrium that, importantly to mention, does render Lyapunov function (7) to be positive, is $\|\mathbf{x}-\overline{\mathbf{x}}\|_{\min }=0.0334$. This is another conservative result yet since it is sufficient, it is greater than the instability bound $r_{x}$, as expected.

In Fig. 3, phase diagrams for the case when $V_{2}=14.8 \mathrm{~V}$ and when the initial system values are chosen to be $\mathbf{x}_{0}=$ $[1.116 \mathrm{~V}-0.665 \mathrm{~V} 27.442 \mathrm{~mA}]^{\top}$, are depicted. From Fig. 3, it can be easily envisioned that the phase portrait lies inside the sphere with radius $K=53.6594$, which bounds system trajectories. It is less easy to envision, yet it has been checked, that the chaotic trajectory for the smaller values of the state variables lies in the region where the instability Lyapunov function is positive yet greater than the instability bound $r_{x}$.

\section{CONClusion}

In this paper, Lyapunov analyses are provided that characterize a bounded set in which the chaotic behavior of the Colpitts oscillator is contained. The analysis is validated by an illustrative simulations. The future research will include polytopic Lyapunov functions in order to obtain tighter bounds on the chaotic motion of the oscillator.

\section{APPENDIX}

The obtained parameters for the transistor's large signal model are $I_{S}=47.1 \mathrm{pA}$ and $\eta=0.7894$. Other circuit parameters are: $R=20 \Omega, R_{E}=510 \Omega, C_{1}=10 \mathrm{pF}$, $C_{2}=10 \mathrm{pF}, L=10 \mathrm{nH}, \beta=100, V_{T}=0.026 \mathrm{~V}$, and $V_{1}=1 \mathrm{~V}$. Voltage of the voltage generator $V_{2}$ is varied in the range from $1 \mathrm{~V}$ to $15 \mathrm{~V}$.

\section{ACKNOWLEDGMENTS}

This material is based upon work supported by the National Science Foundation under Grant No. 1528036 (NRI: ASPIRE: Automation Supporting Prolonged Independent Residence for the Elderly). The work was also supported by the Republic of Serbia Ministry of Science project TR33020. 


\section{REFERENCES}

[1] M. P. Kennedy, "Chaos in the Colpitts oscillator," IEEE Transactions on Circuits and Systems I: Fundamental Theory and Applications, vol. 41, no. 11, pp. 771-774, 1994.

[2] L. O. Chua, C. W. Wu, A. Huang, and G.-Q. Zhong, "A universal circuit for studying and generating chaos. i. routes to chaos," IEEE Transactions on Circuits and Systems I: Fundamental Theory and Applications, vol. 40, no. 10, pp. 732-744, 1993.

[3] L. Chua, "A universal circuit for studying and generating chaos-part ii: Stange attractors," IEEE Trans. Circuits Syst. I, vol. 40, no. 10, pp. $182-186,1993$.

[4] G. M. Maggio, O. De Feo, and M. P. Kennedy, "Nonlinear analysis of the Colpitts oscillator and applications to design," IEEE Transactions on Circuits and Systems I: Fundamental Theory and Applications, vol. 46, no. 9, pp. 1118-1130, 1999.

[5] G. M. Maggio, M. Di Bernardo, and M. P. Kennedy, "Nonsmooth bifurcations in a piecewise-linear model of the Colpitts oscillator," IEEE Transactions on Circuits and Systems I: Fundamental Theory and Applications, vol. 47, no. 8, pp. 1160-1177, 2000.

[6] O. De Feo, G. M. Maggio, and M. P. Kennedy, "The Colpitts oscillator: Families of periodic solutions and their bifurcations," International journal of bifurcation and chaos, vol. 10, no. 05, pp. 935-958, 2000.

[7] A. Čenys, A. Tamaševičius, A. Baziliauskas, R. Krivickas, and E. Lindberg, "Hyperchaos in coupled Colpitts oscillators," Chaos, Solitons \& Fractals, vol. 17, no. 2-3, pp. 349-353, 2003.

[8] J. Kengne, J. Chedjou, G. Kenne, and K. Kyamakya, "Dynamical properties and chaos synchronization of improved Colpitts oscillators," Communications in Nonlinear Science and Numerical Simulation, vol. 17, no. 7, pp. 2914-2923, 2012.

[9] T. Jiang, S. Qiao, Z.-G. Shi, L. Peng, J. Huangfu, W.-Z. Cui, W. Ma, and L.-X. Ran, "Simulation and experimental evaluation of the radar signal performance of chaotic signals generated from a microwave Colpitts oscillator," Progress In Electromagnetics Research, vol. 90, pp. 15-30, 2009.

[10] Z.-G. Shi, S. Qiao, K. S. Chen, W.-Z. Cui, W. Ma, T. Jiang, and L.-X. Ran, "Ambiguity functions of direct chaotic radar employing microwave chaotic Colpitts oscillator," Progress In Electromagnetics Research, vol. 77, pp. 1-14, 2007.

[11] U. Parlitz and L. Junge, "Synchronization of chaotic systems," in Control Conference (ECC), 1999 European. IEEE, 1999, pp. 4637-4642.

[12] G. H. Li, S. P. Zhou, and K. Yang, "Controlling chaos in Colpitts oscillator," Chaos, Solitons \& Fractals, vol. 33, no. 2, pp. 582-587, 2007.

[13] G. Leonov, "Strange attractors and classical stability theory," Nonlinear dynamics and systems theory, vol. 8, no. 1, pp. 49-96, 2008.

[14] H. K. Khalil, Nonlinear Systems, 3rd ed. Upper Saddle River, NJ: Prentice Hall, 2002.

[15] D. D. Siljak, Decentralized Control of Complex Systems. Courier Corporation, 2011. 